\title{
Co-infection Model Formulation to Evaluate the Transmission Dynamics of Malaria and Dengue Fever Virus
}

\author{
${ }^{*}$ OLUWAFEMI, TJ; ${ }^{2}$ AKINWANDE, NI; ${ }^{2}$ OLAYIWOLA, RO; ${ }^{3}$ KUTA, AF; \\ ${ }^{4}$ AZUABA, E
}

\author{
${ }^{*}$ General Studies Department, Newgate College of Health Technology, Minna, Niger State, Nigeria \\ ${ }^{2}$ Department of Mathematics, Federal University of Technology, Minna, Niger State, Nigeria \\ ${ }^{3}$ Department of Microbiology, Federal University of Technology, Minna, Nigeria \\ ${ }^{4}$ Department of Mathematics, Bingham University, Karu, Nasarawa State, Nigeria \\ *Corresponding Author Email: dayofemi985@gmail.com; Tel: +234(0)8138066838
}

\begin{abstract}
A mathematical model of the co-infection dynamics of malaria and dengue fever condition is formulated. In this work, the Basic reduction number is computed using the next generation method. The diseasefree equilibrium (DFE) point of the model is obtained. The local and global stability of the disease-free equilibrium point of the model is established. The result show that the DFE is locally asymptotically stable if the basic reproduction number is less than one but may not be globally asymptotically stable.
\end{abstract}

\section{DOI: https://dx.doi.org/10.4314/jasem.v24i7.10}

Copyright: Copyright (C) 2020 Oluwafemi et al. This is an open access article distributed under the Creative Commons Attribution License (CCL), which permits unrestricted use, distribution, and reproduction in any medium, provided the original work is properly cited.

Dates: Received: 16 May 2020; Revised: 29 June 2020; Accepted: 07 July 2020

Keywords: Malaria; Dengue Fever; Co-infection; Basic reproduction number; Disease-Free equilibrium

Malaria is a mosquito-borne disease caused by the Plasmodium parasite, which is transmitted through the bites of an infected anopheles mosquito. Malaria is caused by five different species of Plasmodium: Plasmodium falciparum, Plasmodium malariae, Plasmodium ovale, Plasmodium vivax, and Plasmodium knowlesi. However, Plasmodium falciparum is the most prevalent in Africa and it causes the highest mortality rate induced by the disease (Olumese P., 2005). The World Health Organization (WHO) world malaria report, an estimated 219 million cases of malaria occurred worldwide in 2017 with Plasmodium falciparum and Plasmodium vivax parasite species posing the extreme public health challenge. In the WHO African Region which has the world's greatest proportions of the population at high menace of malaria, $P$. falciparum is found to be most prevalent and accounts for $99.7 \%$ of estimated malaria cases while $P$. vivax is responsible for $74.1 \%$ of malaria cases in the WHO Region of Americas (WHO, 2018). Dengue is an infectious disease caused by any of the four dengue virus serotypes: DENVs 1-4. It is a mosquito-borne disease and is primarily transmitted to humans by the female Aedes mosquito. Dengue is highly prevalent in tropical and subtropical regions, reflecting the distribution of the vector, Aedes aegypti mosquitoes. Nearly one-third of the global population is at risk for infection (Messina et al., 2014). Infection with DENV results in varying degrees of pathological conditions, ranging from mild asymptomatic dengue fever (DF) to severe dengue hemorrhagic fever (DHF) and dengue shock syndrome (DSS) which may turn fatal (Murphy and Whitehead, 2011). Infected humans are the main carriers and multipliers of the virus, serving as a source of the virus for uninfected mosquitoes. Patients who are already infected with the dengue virus can transmit the infection (for 4-12 days) via Aedes mosquitoes after their first symptoms appear. When a person recovers from dengue infection they develop long-term immunity to that specific virus, but not the other three dengue viruses. If the person becomes infected again with a different dengue virus, there is an increased chance that they may develop into dengue hemorrhagic fever (Nyerere et al., 2017). Dengue cannot be spread from human to human. Dengue and malaria are the most prevalence arthropod-borne diseases with an estimated global incidence of 390 million and 214 million cases a year, respectively (Chong et al., 2017). Dengue viral and malaria parasitic co-infection in an individual is regarded as a 'severe malaria' case (Rao et al., 2016). Mutua et al. (2015) developed a mathematical model to describe the co-infection dynamics of malaria and typhoid. Elmojtaba (2016) formulated a mathematical model to study the co-infection dynamics of malaria and visceral leishmaniasis. Bakare and Nwozo (2016) 
developed and analyzed a mathematical model to explore malaria and schistosomiasis confection. Mensah et al. (2018) in their work proposed a mathematical model to study the transmission dynamics of Zika and Malaria in the malaria-endemic area. Aldia and Agustin (2018) formulated a mathematical model to understand the spread of dengue and chikungunya confection in a closed population. In this work, we proposed a mathematical model for the Co-infection dynamics of malaria and dengue fever.

\section{MATERIALS AND METHODS}

Model Formulation: In this model, the total human population at any given time $t$ denoted by $N_{h}$ is divided into eight sub-classes which are susceptible humans $\left(S_{h}\right)$, individuals exposed to malaria only $\left(E_{h m}\right)$, individuals infected with malaria only $\left(I_{h m}\right)$ , individuals exposed to dengue fever only $\left(E_{h d}\right)$, individuals infected with only dengue fever $\left(I_{h d}\right)$, individuals exposed to malaria and dengue fever coinfection $\left(E_{m d}\right)$, individuals infected with malaria and dengue fever co-infection $\left(I_{m d}\right)$, individuals that recovered from malaria and dengue fever $\left(R_{h}\right)$. The vector population includes the Malaria Parasite noncarrier vectors $\left(S_{m}\right)$, Malaria parasite carrier vectors $\left(I_{m}\right)$, Dengue virus non-carrier vectors $\left(S_{d}\right)$, and Dengue fever carrier vectors $\left(I_{d}\right)$. Susceptible individuals are recruited through constant $\Lambda_{h}$. Susceptible individuals are infected with dengue fever at through contact with the infectious mosquito at a rate $\alpha_{d}$, infected with malaria at a rate $\alpha_{m}$, individuals who recover from malaria returns to the susceptible class at a rate of $\gamma_{h}$, a susceptible individual have a natural death rate of $\mu_{h}$. The class of individuals exposed to malaria only are generated by susceptible individuals infected with malaria only and reduced by the rate of contacting dengue fever at a rate $\alpha_{d}$, the rate of progression to malaria only infected class $\kappa_{1}$ and natural death rate $\mu_{h}$. The class of individuals infected with malaria $\left(I_{h m}\right)$ is increased by $\kappa_{1}$ the rate of progression from malaria exposed class, reduced by the rate of contacting dengue fever at a rate $\alpha_{d}$, disease-induced death rate $\delta_{1}$, malaria only recovery rate $\theta_{1}$, and natural death rate $\mu_{h}$. Individuals that are exposed to dengue fever only are generated by susceptible individuals infected with dengue fever at a rate $\alpha_{d}$, reduced by the natural death rate $\mu_{h}$, rate of progression to infected class for dengue fever only at the rate, $\kappa_{2}$ and the rate at which susceptible individuals contact malaria only. The population of individuals with dengue fever only $\left(I_{h d}\right)$ is generated by individuals that progressed from the exposed class $\left(E_{h d}\right)$ at the rate $\kappa_{2}$. It is also reduced by disease-induced death rate $\delta_{2}$, recovery rate from dengue fever only $\theta_{2}$ and the rate of contacting malaria only. The population of individuals exposed to malaria and dengue fever co-infection $\left(E_{m d}\right)$ is increased by the rate of acquiring malaria through contact with the parasite carrier vectors and dengue fever through contact with dengue virus carrier vectors but reduced by natural death rate and rate of progression to infected malaria and dengue fever coinfection class $\kappa_{3}$. Infected malaria and dengue fever co-infection class $\left(I_{m d}\right)$ are increased by $\kappa_{3}$ and reduced by the natural death rate, co-infection recovery rate $\theta_{3}$, and disease-induced death rate $\delta_{3}$. The recovery class $\left(R_{h}\right)$ is generated by the individuals who recovery from malaria only at the rate $\theta_{1}$, individuals who recover from dengue fever only at the rate $\theta_{2}$, individuals who recover from both diseases at the rate $\theta_{3}$, and reduced by natural death rate and individuals who return to susceptible class after recovery at the rate $\gamma_{h}$. The Malaria parasite non-carrier vector population $\left(S_{m}\right)$ is generated by a constant $\Lambda_{m}$, reduced by the vector natural death rate $\mu_{m}$ and the rate at which the non-carrier vector acquires malaria parasite through contact with exposed and infected individuals with malaria only and co-infection of malaria and dengue fever given as $\alpha_{v m}$ The Malaria parasite carrier vector population is generated by the rate at which the non-carrier vector acquires malaria through contact with exposed and infected individuals with malaria only and coinfection of malaria and dengue fever and the natural death rate $\mu_{m}$. The Dengue virus non-carrier vector 
population $\left(S_{d}\right)$ is generated by a constant $\Lambda_{d}$, reduced by the vector natural death rate $\mu_{d}$, and the rate at which the Dengue virus non-carrier vector acquires dengue virus through contact with exposed and infected individuals with dengue fever only and co-infection of malaria and dengue fever given as $\alpha_{v d}$

The Dengue virus carrier vector class $\left(I_{d}\right)$ is increased by the rate at which the Dengue virus noncarrier vector acquires dengue virus through contact with exposed and infected individuals with dengue fever only and co-infection of malaria and dengue fever and reduced by the vectors natural death rate $\mu_{d}$.

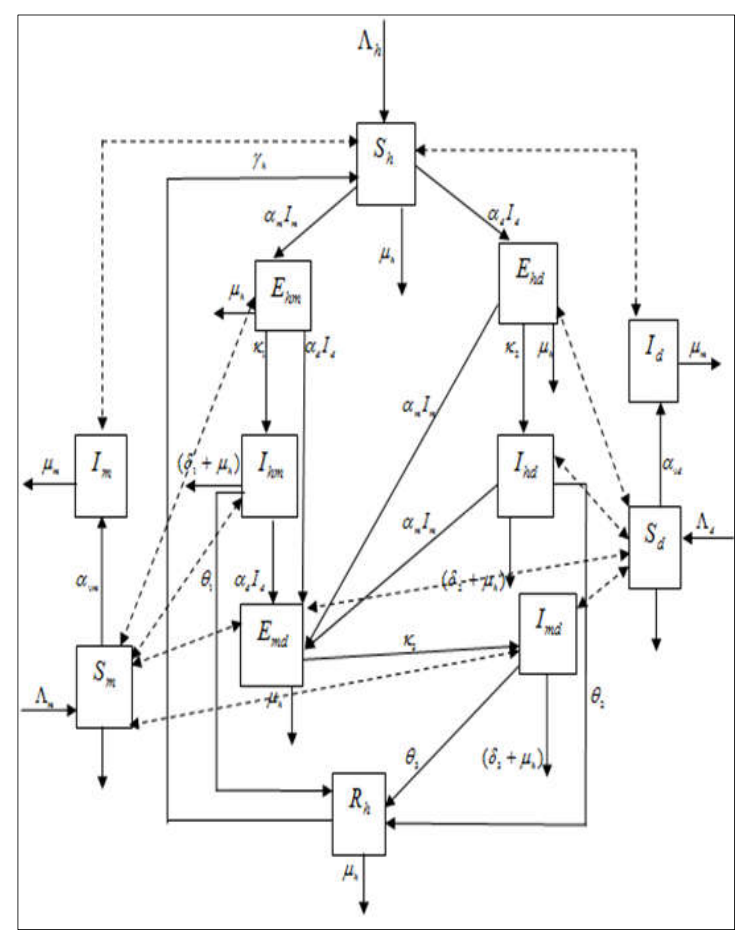

Fig 1: Schematic Representation of the Model

Assumptions of the Model: The following Assumptions are made in formulating the model: (i) Recruitment into the susceptible population is constant (ii) The recovery population include those jointly infected with Malaria and Dengue fever only (iii) Recovery from Dengue fever is permanent.

$$
\begin{gathered}
\frac{d E_{m d}}{d t}=\alpha_{d} I_{d} E_{h m}+\alpha_{d} I_{d} I_{h m}+\alpha_{m} I_{m} E_{h d}+\alpha_{m} I_{m} I_{h d}-\left(\kappa_{3}+\mu_{h}\right) E_{m d} \\
\frac{d I_{m d}}{d t}=\kappa_{3} E_{m d}-\left(\delta_{3}+\theta_{3}+\mu_{h}\right) I_{m d} \\
\frac{d R_{h m}}{d t}=\theta_{1} I_{h m}+\theta_{2} I_{h d}+\theta_{3} I_{m d}-\left(\gamma_{h}+\mu_{h}\right) R_{h m} \\
\frac{d I_{m}}{d t}=\Lambda_{m}-\alpha_{v m}\left(E_{h m}+I_{h m}+E_{m d}+I_{m d}\right) S_{m}-\mu_{m} S_{m} \\
\left.\frac{d S_{d}}{d t}=\Lambda_{d}-\alpha_{v d}+E_{m d}+I_{m d}\right) S_{m}-\mu_{m} I_{m} \\
\frac{d I_{d}}{d t}=\alpha_{v d}\left(E_{h d}+I_{h d}+E_{m d}+I_{m d}\right) S_{d}-\mu_{d} I_{d}
\end{gathered}
$$


Table 1: Variables of the Model

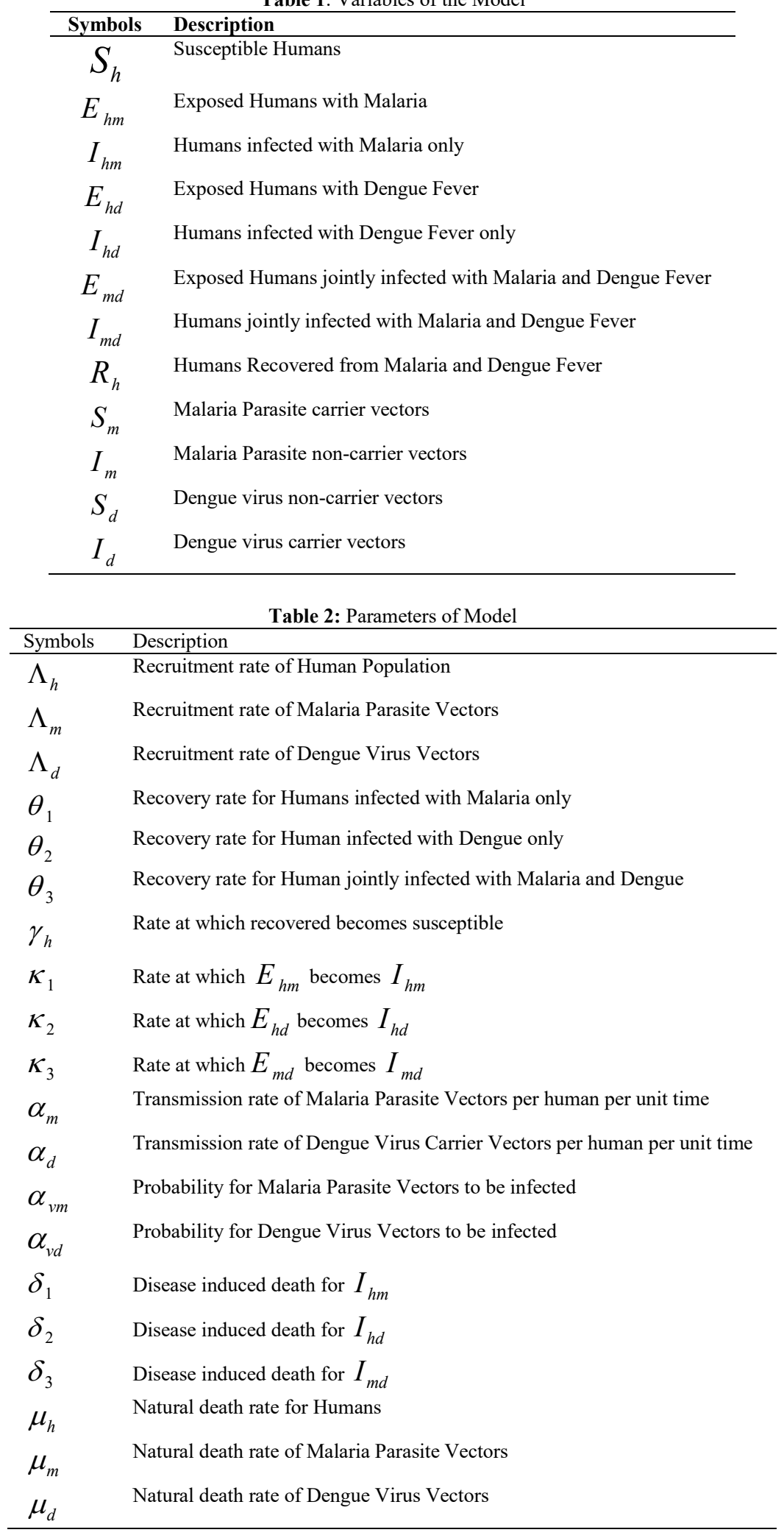

Disease Free Equilibrium (DFE) Point: This is the state solution where is no infection in the population.
The DFE of the model is obtained when the righthand side of the model equation (1) - (12) is set to 
zero. Thus, the DFE point of Malaria-Dengue Fever co-infection is given as in equation 13 .

Basic Reproduction Number: The basic reproduction number is defined as the expected number of secondary infections produced by an index case in a completely susceptible population. For this research, the basic reproduction number is defined as the number of secondary malaria (or dengue) infections due to single malaria (or a single dengue-infective) individual. Applying the next generation method, the basic reproduction number is the spectral radius of the matrix $F V^{-1}$ where $F$ and $V$ are transmission and transition matrices respectively defined as in equation 14.

$$
\begin{aligned}
& \varepsilon_{0}=\left(S_{h}^{0}, E_{h m}^{0}, I_{h m}^{0}, E_{h d}^{0}, I_{h d}^{0}, E_{m d}^{0}, I_{m d}^{0}, R_{h m}^{0}, R_{h d}^{0}, S_{m}^{0}, I_{m}^{0}, S_{d}^{0}, I_{d}^{0}\right) \\
& =\left(\frac{\Lambda_{h}}{\mu_{h}}, 0,0,0,0,0,0,0,0, \frac{\Lambda_{m}}{\mu_{m}}, 0, \frac{\Lambda_{d}}{\mu_{d}}, 0\right) \\
& F=
\end{aligned}
$$

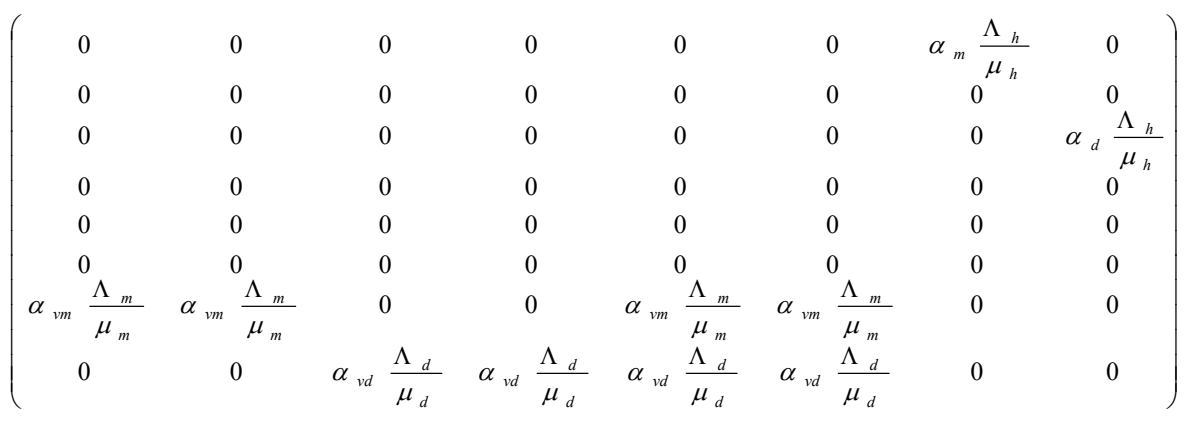

$$
\begin{aligned}
& V=\left(\begin{array}{cccccccc}
\eta_{1} & 0 & 0 & 0 & 0 & 0 & 0 & 0 \\
0 & z_{1} & 0 & 0 & 0 & 0 & 0 & 0 \\
0 & 0 & \eta_{2} & 0 & 0 & 0 & 0 & 0 \\
0 & 0 & 0 & z_{2} & 0 & 0 & 0 & 0 \\
0 & 0 & 0 & 0 & \eta_{3} & 0 & 0 & 0 \\
0 & 0 & 0 & 0 & 0 & z_{3} & 0 & 0 \\
0 & 0 & 0 & 0 & 0 & 0 & \mu_{m} & 0 \\
0 & 0 & 0 & 0 & 0 & 0 & 0 & \mu_{d}
\end{array}\right)
\end{aligned}
$$

The basic reproduction number $R_{0}$ is given as

$$
R_{0}=\max \left\{\sqrt{\frac{\alpha_{m} \alpha_{v m} \Lambda_{h} \Lambda_{m}}{\eta_{1} \mu_{h} \mu_{m}^{2}}}, \sqrt{\frac{\alpha_{d} \alpha_{v d} \Lambda_{h} \Lambda_{d}}{\eta_{2} \mu_{h} \mu_{d}^{2}}}\right\}
$$

\section{RESULT AND DISCUSSION}

Local Stability of the Disease-Free Equilibrium: Theorem: The Disease-Free Equilibrium of the Model Equations (1) - (12) is locally asymptotically stable if $R_{0}<1$ and unstable if otherwise.

Components in are given as
Proof: The system (1) - (12) at DFE is given as

$$
J\left(\varepsilon_{0}\right)=\left(\begin{array}{ll}
A & B
\end{array}\right)
$$


Co-infection Model Formulation to Evaluate the Transmission.....

$$
\begin{aligned}
& A= \\
& \left(\begin{array}{ccccccc}
-\mu_{h} & 0 & 0 & 0 & 0 & 0 & 0 \\
0 & -\eta_{1} & 0 & 0 & 0 & 0 & 0 \\
0 & \kappa_{1} & -z_{1} & 0 & 0 & 0 & 0 \\
0 & 0 & 0 & -\eta_{2} & 0 & 0 & 0 \\
0 & 0 & 0 & \kappa_{2} & -z_{2} & 0 & 0 \\
0 & 0 & 0 & 0 & 0 & -\eta_{3} & 0 \\
0 & 0 & 0 & 0 & 0 & \kappa_{3} & -z_{3} \\
0 & 0 & \theta_{1} & 0 & \theta_{2} & 0 & \theta_{3} \\
0 & -\alpha_{v m} \frac{\Lambda_{m}}{\mu_{m}} & -\alpha_{v m} \frac{\Lambda_{m}}{\mu_{m}} & 0 & 0 & -\alpha_{v m} \frac{\Lambda_{m}}{\mu_{m}} & -\alpha_{v m} \frac{\Lambda_{m}}{\mu_{m}} \\
0 & \alpha_{v m} \frac{\Lambda_{m}}{\mu_{m}} & \alpha_{v m} \frac{\Lambda_{m}}{\mu_{m}} & 0 & 0 & \alpha_{v m} \frac{\Lambda_{m}}{\mu_{m}} & \alpha_{v m} \frac{\Lambda_{m}}{\mu_{m}} \\
0 & 0 & 0 & -\alpha_{v d} \frac{\Lambda_{d}}{\mu_{d}} & -\alpha_{v d} \frac{\Lambda_{d}}{\mu_{d}} & -\alpha_{v d} \frac{\Lambda_{d}}{\mu_{d}} & -\alpha_{v d} \frac{\Lambda_{d}}{\mu_{d}} \\
0 & 0 & 0 & \alpha_{v d} \frac{\Lambda_{d}}{\mu_{d}} & \alpha_{v d} \frac{\Lambda_{d}}{\mu_{d}} & \alpha_{v d} \frac{\Lambda_{d}}{\mu_{d}} & \alpha_{v d} \frac{\Lambda_{d}}{\mu_{d}}
\end{array}\right) \\
& \begin{array}{l}
B= \\
\left(\begin{array}{ccccc}
\gamma_{h} & 0 & -\frac{\alpha_{m} \Lambda_{h}}{\mu_{h}} & 0 & -\frac{\alpha_{d} \Lambda_{h}}{\mu_{h}} \\
0 & 0 & \frac{\alpha_{m} \Lambda_{h}}{\mu_{h}} & 0 & 0 \\
0 & 0 & 0 & 0 & 0 \\
0 & 0 & 0 & 0 & \frac{\alpha_{d} \Lambda_{h}}{\mu_{h}} \\
0 & 0 & 0 & 0 & 0 \\
0 & 0 & 0 & 0 & 0 \\
0 & 0 & 0 & 0 & 0 \\
-z_{4} & 0 & 0 & 0 & 0 \\
0 & -\mu_{m} & 0 & 0 & 0 \\
0 & 0 & -\mu_{m} & 0 & 0 \\
0 & 0 & 0 & -\mu_{d} & 0 \\
0 & 0 & 0 & 0 & -\mu_{d}
\end{array}\right)
\end{array}
\end{aligned}
$$

Where $z_{4}=\left(\gamma_{h}+\mu_{h}\right)$

Applying Gauss Jordan elimination method we have the following from the characteristics equation:

$$
\left.\begin{array}{l}
\lambda_{1}=-\mu_{h}<0, \lambda_{2}=-\eta_{1}<0, \lambda_{3}=-z_{1}<0, \lambda_{4}=-\eta_{2}<0, \lambda_{5}=-z_{2}<0, \lambda_{6}=-\eta_{3}<0, \\
\lambda_{7}=-z_{3}<0, \lambda_{8}=-z_{4}<0, \lambda_{9}=-\mu_{m}<0, \lambda_{10}=-\left(\mu_{m}-\frac{\alpha_{v m} \alpha_{m} \Lambda_{m} \Lambda_{h}\left(\kappa_{1}+z_{1}\right)}{\eta_{1} z_{1} \mu_{m} \mu_{h}}\right)<0, \\
\lambda_{11}=-\mu_{d}<0, \lambda_{12}=-\left(\mu_{d}-\frac{\alpha_{v d} \alpha_{d} \Lambda_{d} \Lambda_{h}\left(\kappa_{2}+z_{2}\right)}{\eta_{2} z_{2} \mu_{d} \mu_{h}}\right)<0
\end{array}\right\}
$$

From $\lambda_{10}$ we have

$$
\lambda_{10}=-\left(1-R_{0 m}^{2}\right)<0
$$

It, therefore, implies that $\lambda_{10}<0$ if $R_{0 m}<1$

Also from $\lambda_{12}$ we have

$$
\lambda_{12}=-\left(1-R_{0 d}^{2}\right)<0
$$


It shows that $\lambda_{12}<0$ if $R_{0 d}<1$

Therefore, the model system is locally asymptotically stable at DFE if and only if $R_{0}<1$ i.e. $R_{0 m}<1$ and $R_{0 d}<1$.

Global Stability of the Disease-Free Equilibrium: The global asymptotic stability (GAS) of the disease-free equilibrium is investigated using theorem in [2]. We re-write the model as

$$
\begin{aligned}
& \frac{d X}{d t}=H(X, Z) \\
& \frac{d Z}{d t}=G(X, Z), \quad G(X, 0)=0
\end{aligned}
$$

Where $X=\left(S_{h}, R_{h m}, S_{m}, S_{d}\right)$

and $Z=\left(E_{h m}, I_{h m}, E_{h d}, I_{h d}, E_{m d}, I_{m d}, I_{m}, I_{d}\right)$

With the components $X$ denoting the uninfected population and components $Z$ denoting the infected population.

The disease-free equilibrium is now denoted as

$$
E^{0}=\left(X^{*}, 0\right) ; \quad X^{*}=\left(\frac{\Lambda_{h}}{\mu_{h}}, 0, \frac{\Lambda_{m}}{\mu_{m}}, \frac{\Lambda_{d}}{\mu_{d}}\right)
$$

The following conditions must be satisfied to guarantee global asymptotical stability:

i. $\quad \frac{d X}{d t}=H(X, 0) ; X^{*}$ is globally asymptotically stable

ii. $\quad G(X, Z)=P Z-\hat{G}(X, Z), \hat{G}(X, Z) \geq 0$ for $(X, Z) \in \Omega$,

Where $P=D_{z} G\left(X^{*}, 0\right)$ is a M-matrix (the off-diagonal elements of $\mathrm{P}$ are non-negative) and $\Omega$ is the region where the model makes biological sense.

If the system satisfies the condition above, then the theorem below holds.

Theorem: The fixed point $E^{0}=\left(X^{*}, 0\right)$ is globally asymptotically stable equilibrium of the system provided $R_{0}<1$ that and the conditions in (29) are satisfied.

Proof: From the model system, we have

$H(X, 0)=\left(\begin{array}{c}\Lambda_{h}-\mu_{h} S_{h}+\gamma_{h} R_{h m} \\ 0 \\ \Lambda_{m}-\mu_{m} S_{m} \\ \Lambda_{d}-\mu_{d} S_{d}\end{array}\right)$

$G(X, Z)=P Z-\hat{G}(X, Z)$ 
Co-infection Model Formulation to Evaluate the Transmission.....

$$
\begin{aligned}
& P=\left(\begin{array}{cccccccc}
-\eta_{1} & 0 & 0 & 0 & 0 & 0 & \alpha_{m} S_{h}^{0} & 0 \\
\kappa_{1} & -z_{1} & 0 & 0 & 0 & 0 & 0 & 0 \\
0 & 0 & -\eta_{2} & 0 & 0 & 0 & 0 & \alpha_{d} S_{h}^{0} \\
0 & 0 & \kappa_{2} & -z_{2} & 0 & 0 & 0 & 0 \\
0 & 0 & 0 & 0 & -\eta_{3} & 0 & 0 & 0 \\
0 & 0 & 0 & 0 & \kappa_{3} & -z_{3} & 0 & 0 \\
\alpha_{v m} S_{m}^{0} & \alpha_{v m} S_{m}^{0} 0 & 0 & 0 & \alpha_{v m} S_{m}^{0} & \alpha_{v m} S_{m}^{0} & -\mu_{m} & 0 \\
0 & 0 & \alpha_{v d} S_{d}^{0} & \alpha_{v d} S_{d}^{0} & \alpha_{v d} S_{d}^{0} & \alpha_{v d} S_{d}^{0} & 0 & -\mu_{d}
\end{array}\right) \\
& -\eta_{1} E_{h m}+\alpha_{m} S_{h}^{0} I_{m} \\
& \kappa_{1} E_{h m}-z_{1} I_{h m} \\
& -\eta_{2} E_{h d}+\alpha_{d} S_{h}^{0} I_{d} \\
& \kappa_{2} E_{h d}-z_{2} I_{h d} \\
& \text { - } \eta_{3} E_{m d} \\
& \kappa_{3} E_{m d}-z_{3} I_{m d} \\
& \alpha_{v m} S_{m}^{0}\left(E_{h m}+I_{h m}+E_{m d}+I_{m d}\right)-\mu_{m} I_{m} \\
& \left.\alpha_{v d} S_{d}^{0}\left(E_{h d}+I_{h d}+E_{m d}+I_{m d}\right)-\mu_{d} I_{d}\right) \\
& G(X, Z)=\left(\begin{array}{c}
\alpha_{m} S_{h} I_{m}-\left(\eta_{1}+\alpha_{d} I_{d}\right) E_{h m} \\
\kappa_{1} E_{h m}-\left(z_{1}+\alpha_{d} I_{d}\right) I_{h m} \\
\alpha_{d} S_{h} I_{d}-\left(\eta_{2}+\alpha_{m} I_{m}\right) E_{h d} \\
\kappa_{2} E_{h d}-\left(z_{2}+\alpha_{m} I_{m}\right) I_{h d} \\
\alpha_{d} I_{d}\left(E_{h m}+I_{h m}\right)+\alpha_{m} I_{m}\left(E_{h d}+I_{h d}\right)-\eta_{3} E_{m d} \\
\kappa_{3} E_{m d}-z_{3} I_{m d} \\
\alpha_{v m} S_{m}\left(E_{h m}+I_{h m}+E_{m d}+I_{m d}\right)-\mu_{m} I_{m} \\
\alpha_{v d} S_{d}\left(E_{h d}+I_{h d}+E_{m d}+I_{m d}\right)-\mu_{d} I_{d}
\end{array}\right) \\
& \hat{G}(X, Z)=\left(\begin{array}{c}
\hat{G}_{1}(X, Z) \\
\hat{G}_{2}(X, Z) \\
\hat{G}_{3}(X, Z) \\
\hat{G}_{4}(X, Z) \\
\hat{G}_{5}(X, Z) \\
\hat{G}_{6}(X, Z) \\
\hat{G}_{7}(X, Z) \\
\hat{G}_{8}(X, Z)
\end{array}\right)=\left(\begin{array}{c}
\alpha_{m} I_{m}\left(S_{h}^{0}-S_{h}\right)+\alpha_{d} I_{d} E_{h m} \\
\alpha_{d} I_{d} I_{h m} \\
\alpha_{d} I_{d}\left(S_{h}^{0}-S_{h}\right)+\alpha_{m} I_{m} E_{h d} \\
\alpha_{m} I_{m} I_{h d} \\
-\left(\alpha_{d} I_{d}\left(E_{h m}+I_{h m}\right)+\alpha_{m} I_{m}\left(E_{h d}+I_{h d}\right)\right) \\
0 \\
\alpha_{v m} S_{m}\left(E_{h m}+I_{h m}+E_{m d}+I_{m d}\right)\left(S_{m}^{0}-S_{m}\right) \\
\alpha_{v d} S_{d}\left(E_{h d}+I_{h d}+E_{m d}+I_{m d}\right)\left(S_{d}^{0}-S_{d}\right)
\end{array}\right)
\end{aligned}
$$

From (35), it shows $\hat{G}_{5}(X, Z)<0$ so the conditions are not met. Therefore, $E^{0}$ may not be globally asymptotically stable when $R_{0}<1$.

Conclusion: In this work, a co-infection model is formulated to study the transmission dynamics of Malaria and Dengue fever virus. The basic reproduction number of the model is derived using the next generation method, local and global stability of the Disease-free equilibrium (DFE) is carried out. It was observed that the DFE is locally asymptotically stable if $R_{0}<1$ but may not be globally asymptotically stable.

\section{REFERENCES}

Aldila, D; Agustin, MR. (2018). "A Mathematical Model of Dengue-Chikungunya Co-Infection in a 
Closed Population". Journal of Physics: Conf. Series DOI :10.1088/1742-6596/974/1/012001

Bakare, EA; Nwozo, CR. (2016). "Mathematical Analysis of Malaria-Schistosomiasis Co-infection model".Epidemiology Research International.. Article ID 3854902, 19 pages. Hindawi Publishing http://dx.doi.org/10.1155/2016/3854902

Castillo-Chavez, C; Blower, S; Van den Driessche, P; Kirschner. D; Abdul-Aziz, Y. (2002). Mathematical Approaches for Emerging and Reemerging Infectious Diseases: Models, Methods and Theory.

Chong, SE; Mohamad, ZRH; Suraiya, S; Lee, KT; Lim, JA; Malar, J. (2017). "The danger of accepting a single diagnosis: case report of concurrent Plasmodium Knowlesi malaria and dengue infection". Malaria Journal, 16:2

Driessche, VDP; Watmough, J. (2002). "Reproduction Numbers and Sub-threshold Endemic Equilibria for Compartmental Models of Disease Transmission". Mathematical Biosciences 180 (12): $29-48$.

Diekmann, O; Heesterbeek, JAP; Metz, JAJ. (1990). "On the definition and computation of Basic Reproduction Ratio in Models for infectious Diseases in Heterogeneous Population". Journal of Mathematical Biology 28 (4): 365-382

Epelboin, L; Hanf, M; Dussart, P; Ouar-Epelboin, S; Djossou, F; Nacher, M; Carme, B. (2012). "Is dengue and malaria co-infection more severe than single infections? A retrospective matched-pair study in French Guiana". Malaria Journal. 11: (142) 1-8.

Idoko, MO; Ado, SA; Umoh, VJ. (2015). "Prevalence of Dengue Virus and Malaria in Patients with Febrile Complaints in Kaduna Metropolis, Nigeria". British Microb. Res. J. 8(1): 343-347
Messina, JP et al. A global compendium of human dengue virus occurrence. Sci. Data 1, 140004 (2014).

Murphy, BR; Whitehead, SS. (2011). "Immune response to dengue virus and prospects for a vaccine". Annual Review of Immunology, vol. 29, pp. 587-619

Mutua, JM; Wang, F; Vaidya, NK. (2015). "Modeling malaria and typhoid fever co-infection dynamics". Mathematical Biosciences, 264: 128144

Niyati, K; Ira, K. (2016). "Dengue Fever: Causes, Complications, and Vaccine Strategies". Journal of Immunology Research. Article ID 6803098, 14 pages. Hindawi Publishing Corporationhttp://dx.doi.org/10.1155/2016/6803 $\underline{098}$

Nyerere, N; Matofali, AX; Mpeshe, SC; Edward, S. (2017). "Modeling the impact of vertical transmission in vectors on the dynamics of dengue fever". World Journal of Modelling and Simulation, 13(3), 219-227.

Olumese, P. (2005)" Epidemiology and surveillance: changing the global picture of malaria-myth or reality?" Acta Tropica 95(3): 265-269.

Rao, MRK; Rabindra, NP; Manoj, KD. (2016)." Prevalence of dengue viral and malaria parasitic co-infections in an epidemic district, Angul of Odisha, India: Aneco-epidemiological and crosssectional study for the prospective aspects of public health". Journal of Infection and Public Health 9, 421-428.

World Health Organization (2018), World Malaria Report, Geneva, Press. 